\title{
Fluorescein angiography of the hereditary choroidal dystrophies
}

\author{
KENNETH G. NOBLE, RONALD E. CARR, AND IRWIN M. SIEGEL \\ From the Department of Ophthalmology, New York University Medical Center, 550 First Avenue, New York, \\ New York 10016
}

SUMMARY The hereditary choroidal dystrophies are divided into (1) geographic choroidal dystrophies (central areolar, peripapillary, generalised), (2) gyrate atrophy, and (3) choroideremia. Each of these disorders is discussed with regard to mode of inheritance, age of onset, symptoms, fundus appearance, and visual function testing. A typical case history of each disorder is presented together with fluorescein angiography, and the fluorescein angiographic findings are related to our present understanding of these diseases.

Fluorescein angiography was found to be most helpful in diagnosing the early cases, by confirming the absence of the choriocapillaris, and in demonstrating either a local or general abnormality. The role of fluorescein angiography in understanding the aetiology of choroidal dystrophies is discussed.

The hereditary choroidal dystrophies can be classified in the following manner: (1) the geographical choroidal dystrophies, which can be further subdivided into $(a)$ central aerolar choroidal dystrophy, (b) peripapillary choroidal dystrophy, and $(c)$ generalised choroidal dystrophy; (2) gyrate atrophy; and (3) choroideremia. Each of these disorders has a particular mode of inheritance (Table 1). In the early and middle stages of each disease there is usually a characteristic fundus appearance, and this, together with the appropriate family history, is usually sufficient to make the correct diagnosis. However, in the earliest stages of these dystrophies the fundus change may not be as obvious. It is in these cases that fluorescein angiography provides a great deal of helpful information.

This paper describes the role of fluorescein angiography in diagnosing and in understanding the hereditary choroidal dystrophies.

\section{Methods and materials}

From a large group of patients with choroidal disease a group was selected which represented different aspects of the hereditary choroidal dystrophies. All patients were seen at the NYU-Bellevue

Supported in part by Grant EY00213 from the National Eye Institute, National Institutes of Health, Bethesda, Maryland.

Address for reprints: Dr Ronald E. Carr, Department of Ophthalmology, New York University Medical Center, 550 First Avenue, New York, New York 10016.
Retinal Clinic and underwent complete ophthalmological examination.

All patients had fluorescein angiography, and certain psychophysical and electrophysiological diagnostic tests (Goldmann visual fields, electroretinography (ERG), electro-oculography (EOG), and retinal profiles) were performed where indicated. The procedures followed in this laboratory for such testing are fully outlined in previous papers (Carr et al., 1966a; Carr et al., 1966b).

\section{Results}

1. GEOGRAPHIC CHOROIDAL DYSTROPHY This group of disorders is subdivided into three separate entities based on the geographical distribution. The areas which appear ophthalmoscopically normal are functionally normal as measured by psychophysical and electrophysiological testing.

\section{A. Central areolar choroidal dystrophy}

Central areolar choroidal dystrophy is inherited in an autosomal dominant manner (Sandvig, 1955;

\section{Table 1 Hereditary choroidal dystrophies}

\footnotetext{
I. Geographic choroidal dystrophy

$A$. Central areolar choroidal dystrophy (autosomal dominant)

$B$. Peripapillary choroidal dystrophy (autosomal recessive)

C. Generalised choroidal dystrophy (autosomal dominant)

II. Gyrate atrophy (autosomal recessive)

III. Choroideremia (X-linked recessive)
} 

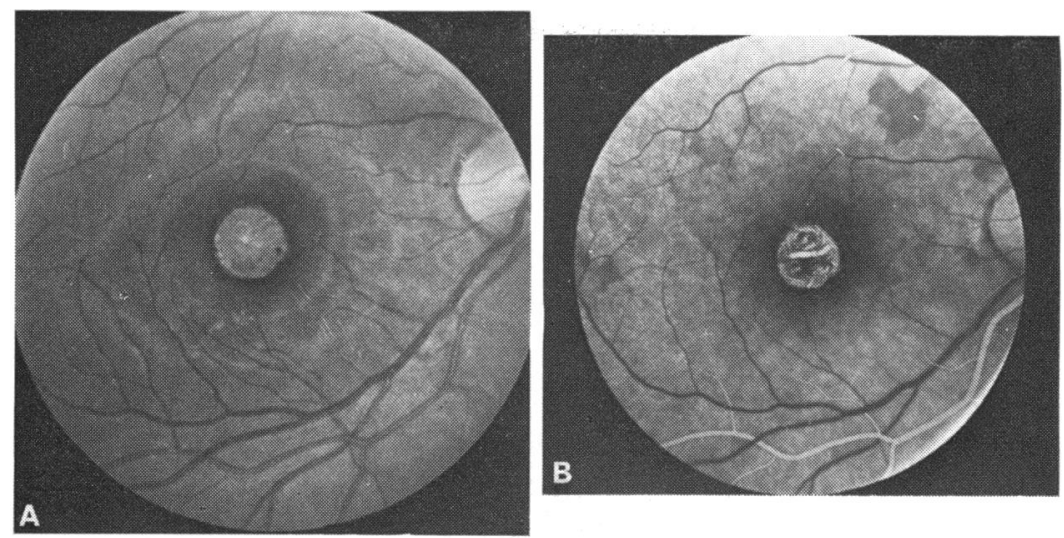

Fig. 1 (Noble, Carr, and Siegel) (case 1) Central AREOLAR CHOROIDAL DYSTROPHY. (A) Wellcircumscribed, circular macular lesion with a central tapetal 'sheen' surrounded by $a$ heaping of pigment. (B)
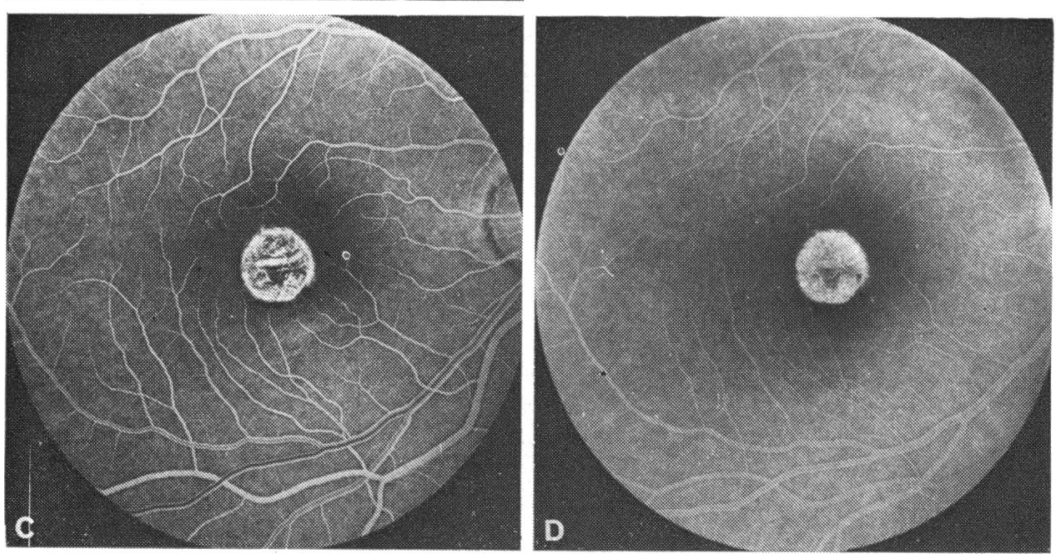
Arterial phase-large choroidal vessel traversing central macular lesion and surrounded by a few smaller choroidal vessels. (C) Mid venous phase; smaller choroidal vessels more easily seen. (D) Late phase; hyperfluorescence of the macula due to leakage from adjacent normal choriocapillaris

Carr, 1965), though autosomal recessive cases (Waardenberg, 1952; Sorsby and Crick, 1953) have been occasionally reported. The onset is in the late second decade to the early fourth decade, with the initial symptom of diminished central visual acuity related to the macular location.

The lesion occupies the central macular area of each eye and is solitary, circumscribed, and circular or ovoid in shape. Although it may increase in size and become irregular in shape, it does not involve the peripapillary region or extend beyond the temporal vessel arcades.

The early fundus picture includes a mottling of the pigment epithelium or a tapetal 'sheen'. At this stage the underlying choroid may appear ophthalmoscopically normal, but with the subsequent loss of pigment epithelium the underlying choroidal vessels are more easily seen. They may be orange-red or yellowish-white in colour. With the continued loss of pigment and choroidal tissue the larger choroidal vessels are more easily seen. In the final stages the sclera is visible with the choroidal vessels coursing above.
Psychophysical and electrophysiological tests confirm that this is not a generalised disorder but is restricted to the area of the visible lesion. The peripheral visual fields are full and a central scotoma can be demonstrated. Dark-adapted final thresholds are elevated in the macular area but are normal beyond it. The electroretinogram is either normal or shows a diminished photopic response, and the EOG light rise is normal (Carr, 1965).

\section{Case 1 (Fig. 1)}

This 30-year-old male had a history of decreasing vision in both eyes of three years' duration. He denied any family history of similar eye disease but none of his family, all living in Pakistan, were examined. His parents are distant cousins.

The best corrected vision was 20/80 - OD and $20 / 40$ + OS. Both fundi demonstrated a wellcircumscribed, circular macular lesion which appeared as a central tapetal 'sheen' surrounded by a heaping of pigment. The remainder of the retina was completely normal.

Colour vision testing on the AOHRR plates was 
Fig. 2 (Noble, Carr, and Siegel) (case 2) JUVENILE HEREDITARY MACULAR DEGENERATION. (A) Diffuse macular tapetal reflex. (B) Arterial phase; hyperfluorescence of macula due to underlying pigment defects. Choroidal vessels are not apparent. (C) Early venous phase; increase in the macular hyperfluorescence. (D) Late venous phase; no evidence of fluorescein dye leakage
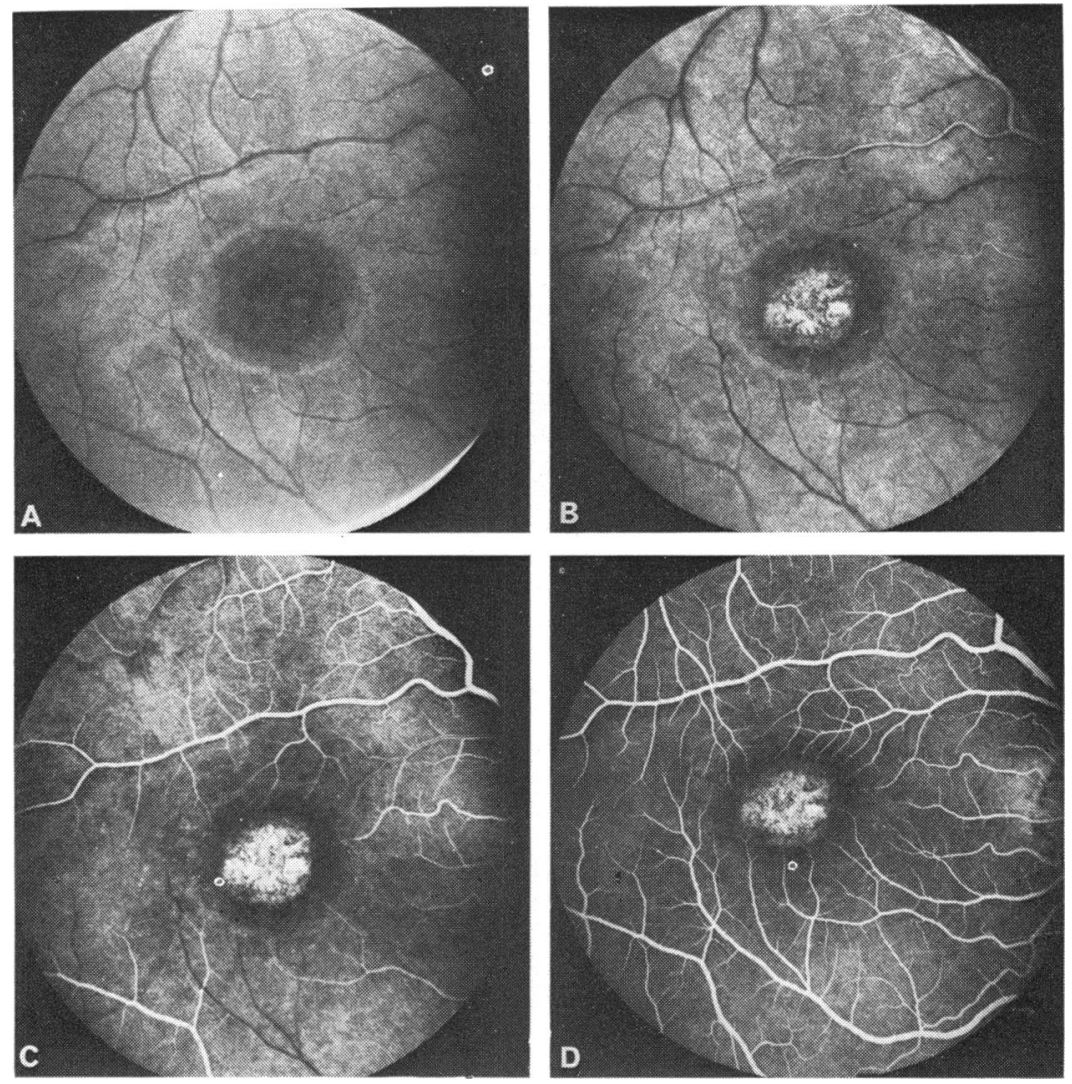

normal. Visual fields on the Goldmann perimeter $\left(0.25\right.$ and $1 \mathrm{~mm}^{2}$ white test objects at full illumination) revealed full peripheral fields and a small $\left(3^{\circ}\right)$ central scotoma in each eye. The electroretinogram and electro-oculographic light rise were normal.

On fluorescein angiography a large choroidal vessel was easily seen traversing the central macular lesion with a few smaller choroidal vessels surrounding it. The vessels filled initially in the choroidal phase and gradually became obscured as fluorescein from adjacent normal choriocapillaris leaked in at the border. The absence of choriocapillaris was confined to the macular lesion and the remainder of the choriocapillaris was normal.

This case represents an early stage of central areolar choroidal dystrophy which presented ophthalmoscopically as a well-circumscribed macular degeneration with a tapetal sheen. Although the choroid beneath the pigment disturbance appeared normal on fundus examination, fluorescein angiography clearly demonstrates the absence of the choriocapillaris.

Compare this fluorescein picture to what is seen in the early stages in another hereditary macular degeneration which may look similar on ophthalmoscopy.

Case 2 (Fig. 2)

This 18-year-old male had a two-year history of decreased vision in both eyes. He denied a family history of similar ocular disorders.

Visual acuity was $20 / 80$ OD and $20 / 200$ OS. The macula of each eye had mild changes in the pigment epithelium in an irregular fashion with the suggestion of a tapetal reflex. Peripheral visual fields were full and an electroretinogram was normal.

A fluorescein angiogram showed hyperfluorescence of dye in the macular region of each eye.

In this case fluorescein angiography revealed a hyperfluorescence of dye due to the increased transmission from the underlying normal choriocapillaris. The defect on fluorescein angiography in this case was only in the retinal pigment epithelium.

\section{B. Peripapillary choroidal dystrophy}

The peripapillary variant of choroidal dystrophy is 


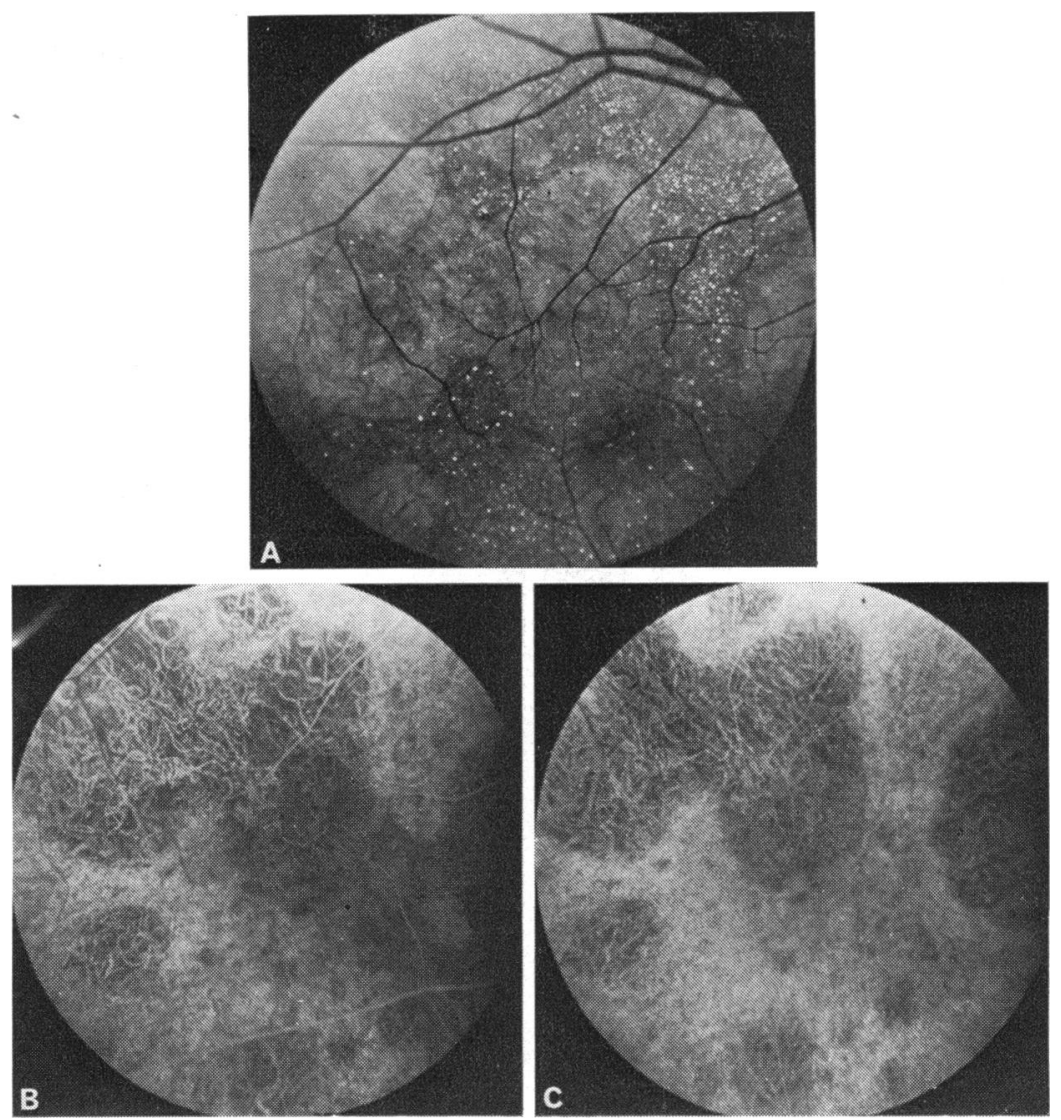

Fig. 3 (Noble, Carr, and Siegel) (case 3)

Peripapillary ChOROIDAL DYSTROPHY. (A) Multiple irregular areas of pigmentary loss. Tiny glistening yellowish-white spots are scattered throughout the posterior pole over normal appearing retina. (B) Mid venous phase; area of pigment loss shows an absent choriocapillaris with the larger choroidal vessels visible.

(C) Late phase; the normal choriocapillaris flush emphasises the contrast with the abnormal choroid in which the large choroidal vessels remain visible inherited as an autosomal recessive (Svensson, 1939; Krill and Archer, 1971) but in many cases a positive family history is lacking. The onset is probably similar to the central areolar variety, although symptoms may occur later when the macula is affected and the vision reduced.

The development of the fundus picture is similar to central areolar choroidal dystrophy, with early changes seen in the retinal pigment epithelium and later an ophthalmoscopically apparent loss of pigment and choroidal tissue. The important distinction between these two disorders is their location. The peripapillary variety begins in the region surrounding the optic disc and slowly enlarges, in finger-like projections, nasally, along the temporal vessels, and into the macula, eventually occupying the entire posterior pole.

Visual fields and dark-adapted final thresholds indicate that peripheral to the involved area retinal function is normal. The electroretinogram is either normal or only slightly reduced, reflecting the extent of the disease (Krill and Archer, 1971; Carr et al., 1975).
Case 3 (Fig. 3)

This 54-year-old male complained of decreased vision in the right eye of three weeks' duration. He denied a family history of any similar ocular disorder, though his parents may have been distant relatives. Family members were not available for examination.

The corrected vision was 20/30 OD and 20/20 - OS. Fundus examination showed multiple irregular areas of pigment epithelial loss in the posterior polar region in both eyes. Over these regions were scattered tiny glistening yellowish-white spots.

Final dark-adaptation thresholds were elevated $3 \log$ units between 3 and $15^{\circ}$ in the superior and temporal quadrants and between 3 and $25^{\circ}$ in the nasal quadrant. More peripheral areas showed normal thresholds. An electroretinogram showed a slight reduction in the photopic and scotopic B-wave amplitude. The EOG light rise was normal.

Fluorescein angiography demonstrated the loss of the pigment epithelium, while beneath these affected regions the choriocapillaris was absent and the choroidal vessels were visible. Adjacent areas appeared completely normal. 
Fig. 4 (Noble, Carr, and Siegel) (case 4) PERIPAPILlaRY PIGMENTARY DYSTROPHY.

(A) Superior temporal vessels of left eye demonstrating irregular ill-defined loss of pigment epithelium. White spots are artifacts. (B) Arterial phase; hyperfluorescence in affected area due to pigment epithelial loss.

(C) Mid venous phase; hyperfluorescence persists and contrasts with normal fluorescein pattern superiorly. A few bone spicules are visible inferiorly.

(D) Peripapillary area of the left eye indicating the distribution of the pigment changes
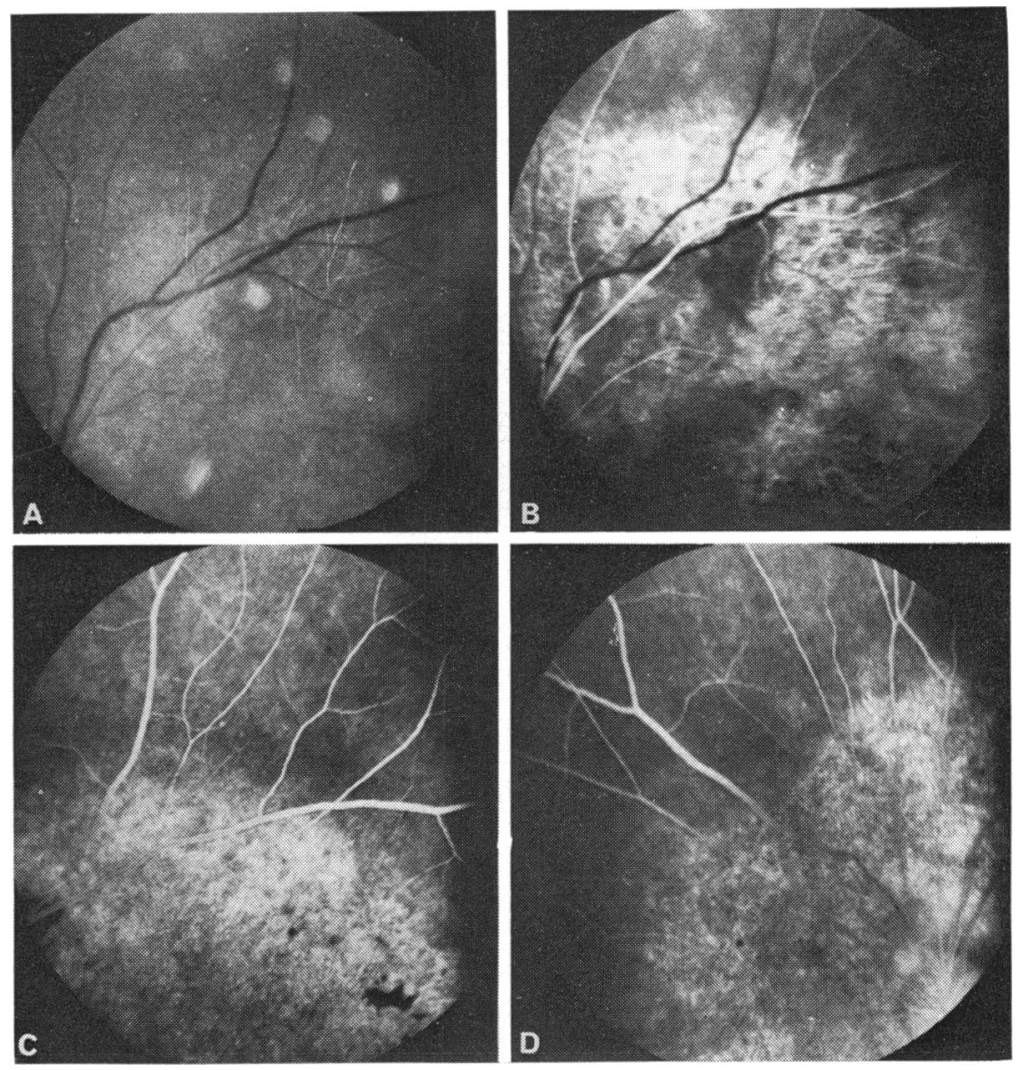

Cases 4, 5, 6 (Fig. 4)

These three siblings, a female aged 53 (case 4), her sister, aged 62 (case 5), and the brother, 66 years old (case 6), gave a similar history of poor central vision since the middle of their third decade. The parents are first cousins and there is a history of consanguinity in both the maternal and paternal grandparents.

The vision in each eye of all three ranged from $10 / 400$ to counting fingers at $1 \mathrm{ft}$. The posterior polar areas showed a generalised loss of the pigment epithelium with irregular borders separating the abnormal central retina from the normal-appearing periphery. Perivascular bone spicule pigmentation and several pigment strands were also seen within the affected regions.

The visual fields performed on cases 5 and 6 demonstrated large, dense central scotoma $\left(64 \mathrm{~mm}^{2}\right.$ white test object) in each eye which corresponded to the fundus area involved. The peripheral fields were full or showed only a slight depression. The electroretinographic findings were variable $-\mathrm{a}$ slight reduction in the scotopic amplitude and a normal photopic response (case 4), a small photopic response without a scotopic increment (case 5), and an absent response (case 6).

Fluorescein angiography, performed in cases 4 and 5, showed a hyperfluorescence in the affected posterior polar region which was due to the retinal pigment epithelium loss, but there was no evidence of choriocapillaris loss. Peripheral areas adjacent to the dye hyperfluorescence were completely normal. Angiographic studies could not be performed in case 6.

The fundus appearance of peripapillary choroidal dystrophy, like central areolar choroidal dystrophy, need not always show obvious, well-defined areas of pigment loss revealing the underlying larger choroidal vessels. The appearance and geographic location of the lesion in case 3 is not dissimilar from that seen in cases 4,5 , and 6 , and functional tests indicated a localisation to the peripapillary and macular area.

Comparison of the fluorescein angiogram, however, revealed significant differences. In case 3 the choriocapillaris and retinal pigment epithelium are absent and the larger choroidal vessels are seen throughout the sequence, whereas in cases 4,5 , and 6 

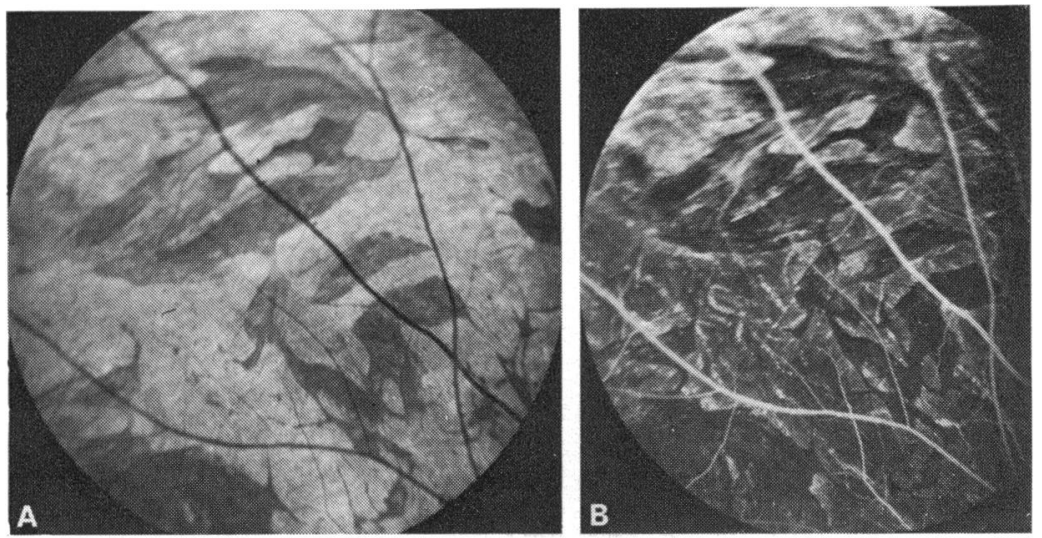

Fig. 5 (Noble, Carr, and Siegel) (cases 7 and 8) GENERALISED CHOROIDAL DYSTROPHY. (A) Representative area of the fundus demonstrating a generalised loss of the pigment epithelium with scattered islands of irregularly shaped pigment accumulation. (B) Fluorescein angiography shows an absence of the choriocapillaris with the larger choroidal vessels
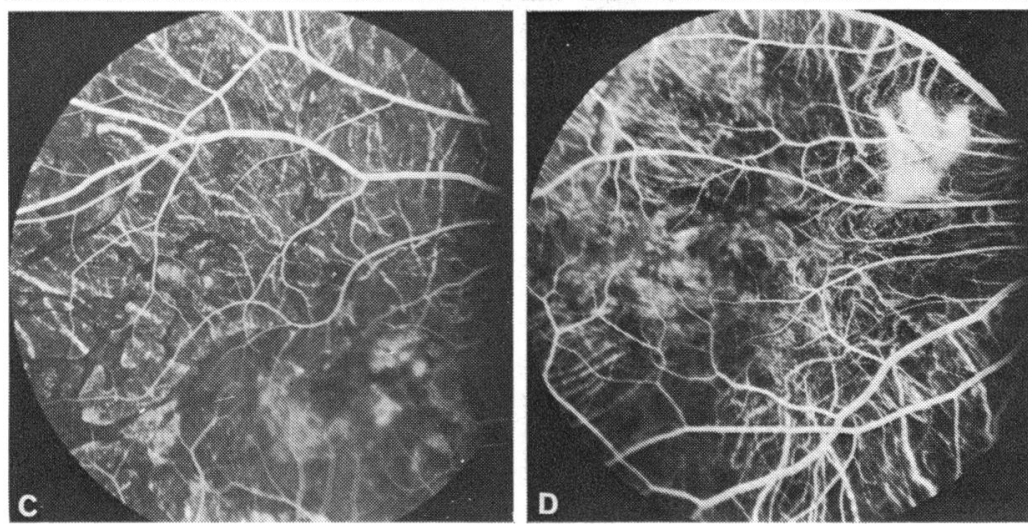
demonstrating calibre irregularities. The islands of pigment accumulation result in hypoftuorescence. (C and D) Fluorescein angiography of the macula of the right eye of cases 7 and 8 respectively demonstrating the profound generalised loss of the choriocapillaris. Gliotic changes are noted in the macula of Fig. $C$. The retinal vessels are normal

the hyperfluorescence of dye is a result of changes in the retinal pigment epithelium only.

\section{Generalised choroidal dystrophy}

This diffuse disorder of the choriocapillaris is usually inherited as an autosomal dominant (Sorsby and Davey, 1955). The onset of symptoms is in the third and fourth decade and is usually manifested subjectively by diminished visual acuity. While symptoms of night blindness may also be present, the subjective changes associated with the photopic system usually predominate.

The early fundus changes include pigment mottling, hypopigmentation, and transparency of the retinal pigment epithelium. Later there is diffuse atrophy of the retinal pigment epithelium and the choroid appears 'sclerotic' with yellowish-white bands. Both the posterior pole and the periphery are involved in varying degrees. Even with diffuse involvement in the more advanced stages the retinal vessels usually remain normal (Franceschetti et al., 1974). In the end stages it cannot be differentiated from other diffuse chorioretinal diseases.

The psychophysical and electrophysiological studies reflect this diffuse involvement. Visual fields reveal a concentric peripheral constriction and the electroretinogram is either subnormal (Franceschetti et al., 1974) or absent (McKay and Spivey, 1962).

Fluorescein angiography performed by Curry and Schonberg (1969) showed a loss of choriocapillaris and visualisation of the choroidal vessels beneath the transparent pigment epithelium. A few scattered areas showed a choroidal flush pattern indicative of some remnants of choriocapillaris.

The following two cases represent an unusual variant of a generalised choroidal dystrophy and show how fluorescein angiography may help in the understanding of certain puzzling disorders which do not fit into our current classifications.

\section{Cases 7 and 8 (Fig. 5)}

Two brothers, aged 11 years (case 7) and 9 years (case 8) have had poor vision since birth. There is no family history of similar ocular disorders or of consanguinity. Two siblings, the parents, and a paternal aunt and uncle were examined and found to be normal. The visual acuity in the older brother was $5 / 200 \mathrm{OU}$ and in the younger brother 5/200 OD 
and 20/200 OS. There was no nystagmus. The fundus appearance of each child was essentially the same. The discs were pink, without evidence of atrophy, and the retinal vessels were normal. Throughout the entire retina there was a loss or thinning of the pigment epithelium associated with irregular bands and clumps of jet black pigment. In some areas there was glial proliferation and chorioretinal atrophy in which the choroidal vessels appeared 'sclerotic'. Scattered throughout were irregularly-shaped islands of pigment epithelial thinning in which the choroid appeared unaffected. The fundus colour was dark greyish-green, unlike the pink colour of unaffected family members. An electroretinogram was not detectable in either child.

The fluorescein angiogram was similar in each case, showing a profound loss of choriocapillaris in the islands of retinal pigment thinning. The choroidal vessels did not fill evenly and showed multiple calibre irregularities. Interspersed between the islands of choriocapillaris loss there was hypofluorescence due to the irregular pigment changes.

The fundus showed a transition from the early thinning of the retinal pigment epithelium, in which the choroid appears normal, to the stages of chorioretinal atrophy, choroidal 'sclerosis', and gliosis. The fluorescein angiogram showed that beneath the transparent retinal pigment epithelium the choriocapillaris was absent and the abnormal choroidal vessels were easily visualised. These findings lend support to the diagnosis of a hereditary congenital generalised choroidal dystrophy.

\section{GYRATE ATROPHY}

Gyrate atrophy is a rare choroidal disease which is inherited as an autosomal recessive (Waardenberg, 1939), although dominant pedigrees have been reported (Franceschetti et al., 1974). The onset of symptoms occurs in the second and third decades and consists of poor night vision and constricted peripheral vision.

The fundus abnormality begins in the midperiphery with a thinning and transparency of the retinal pigment epithelium in which the underlying choroid may appear normal or 'sclerotic'. These areas are typically scalloped in shape and begin as separate isolated areas which may merge to form a garland wreath. The borders have a darker appearance due to pigment accumulation which increases the contrast between the adjacent normal and abnormal tissue.

Progression of the disease leads to pigment clumping, retinal pigment epithelium and choriocapillaris atrophy, and eventual disappearance of the entire choroid exposing the white sclera. The optic nerve and retinal vessels may be normal or abnormal (Krill and Archer, 1971; Franceschetti et al., 1974; Kurstjens, 1965; Duke-Elder, 1966). These changes begin in the midperiphery and slowly extend anteriorly and posteriorly. In the late stages an annular ring of choroidal atrophy may be seen from the periphery to the posterior pole, usually sparing the macula.

The functional tests vary considerably from case to case and seem to be related to the extent of involvement (Franceschetti et al., 1974).

\section{Case 9 (Fig. 6)}

This 12-year-old female with progressive myopia complained of the recent onset of poor night vision and decreasing side vision. There was no significant family history with regard to ocular disorders.

The corrected vision was 20/40 OD and OS with a -9.50 sphere in each eye. In the equatorial regions of both eyes there was a loss of the retinal pigment epithelium in a scalloped fashion. The disc, macula, and retinal vessels were normal. The peripheral visual fields were constricted bilaterally to $30^{\circ}$ $\left(64 \mathrm{~mm}^{2}\right.$ white test object), and an electroretinogram was absent.

Follow-up examination 10 years later revealed an increase in night and peripheral vision symptoms. The best vision was 20/200 OD and 20/50 + OS.

There was slight progression of the equatorial scalloped areas centrally and peripherally, and the sclera was visible in scattered areas. Final darkadaptation thresholds in the left eye showed an elevation of one $\log$ unit above the normal value throughout the horizontal meridian except in the temporal retina at 25 and $30^{\circ}$, where the elevation was 3 to $4 \log$ units above the normal value.

Fluorescein angiography demonstrated the sharp demarcation between normal and abnormal tissue. In the involved scalloped portion the choriocapillaris was absent, while in the peripapillary and macular regions the choriocapillaris flush was seen. In the area separating these two there was a decrease in the transmission of dye due to heavy pigment accumulation.

The fluorescein study in this case is in accord with the functional findings. The scalloped areas of pigment epithelial thinning and loss reveal on fluorescein angiography a profound loss of the choriocapillaris, while adjacent areas without the pigment epithelial disturbances show a normal choriocapillaris flush phase.

\section{CHOROIDEREMIA}

Choroideremia is a generalised degeneration inherited as an X-linked recessive (McCulloch and McCulloch, 1948). The onset of symptoms usually occurs in the first and second decade with night 

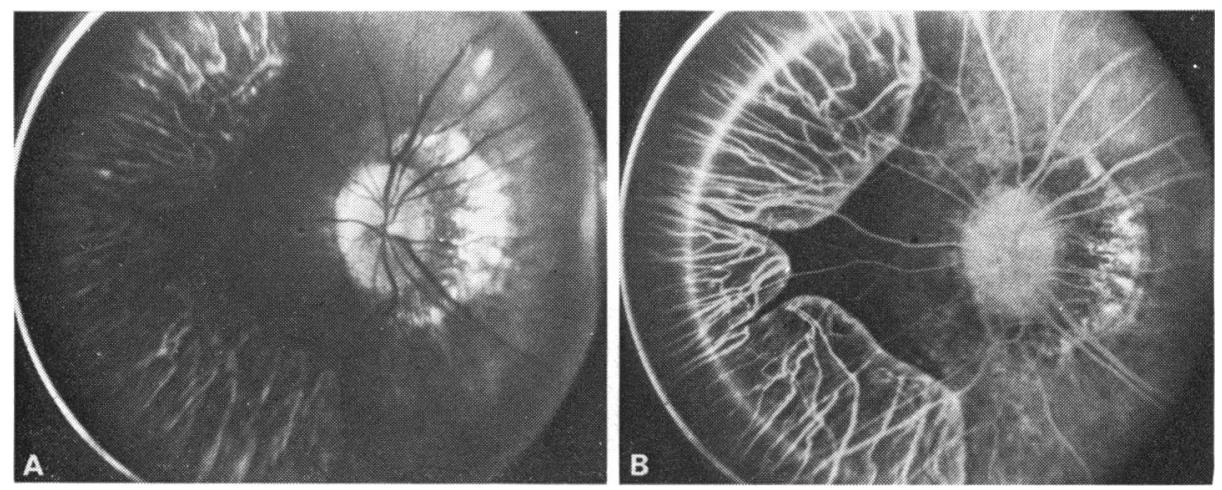

Fig. 6 (Noble, Carr, and Siegel) (case 9) GyRATE ATROPHY. (A) Loss of pigment epithelium in a scalloped pattern revealing underlying white larger choroidal vessels. Peripapillary changes secondary to high myopia are present temporally. (B) Fluorescein angiography demonstrates the sharp demarcation between normal and abnormal tissue. The choriocapillaris is completely absent in the peripheral area while the choriocapillaris flush in other areas is normal. The border is highlighted by hypofluorescence due to pigment accumulation

vision complaints. However, the actual lesion may begin earlier, because a 22-month-old infant with fundus changes has been described (McCulloch and McCulloch, 1948).

The fundus changes in the affected male undergo a typical progression. The initial appearance is a 'salt and pepper' pigment mottling in the equator and posterior pole. At this stage the electroretinogram is abnormal, showing a reduced or absent scotopic component, and final dark-adaptation thresholds are elevated (Franceschetti et al., 1974).

Focal disturbances in the pigment epithelium consisting of pigmentary loss or a metallic sheen follow the 'salt and pepper' mottling and the underlying choroid may appear normal. Occasionally, these areas of focal disturbances assume a shape similar to gyrate atrophy, and the distinction between the two diseases may be difficult (Kurstjens, 1965). Atrophy of the choroid follows with eventual loss of the entire layer and exposure of bare sclera. The rate of progression will vary from individual to individual, and from family to family.

These changes begin in the mid-periphery and progress centrally, the macula being the last affected with central vision preserved until late in the disease. In the final stage the entire fundus shows the diffuse yellowish-white reflex of the sclera.

The carrier female usually has a picture similar to the earliest stage of the affected male (Goodman et al., 1965). Typically there is pigment mottling, best seen in the mid-periphery.

Despite the similarity in appearance of carrier female and early affected males visual function tests (visual fields, final thresholds, electroretinogram) are usually normal in the female (Goodman et al.,
1965). In addition the fundus signs are stationary and visual function tests remain normal (Franceschetti et al., 1974). There are occasional case reports in which the carrier female may have retinal and functional changes similar to the male, but such findings are a rarity (Fraser and Friedmann, 1968; Harris and Miller, 1968).

Case 10 (Fig. $7 a, b$ ) and Case 11 (Fig. 7c,d)

This 35-year-old female (case 10) and her 7-year-old son (case 11) were seen as part of a family study of choroideremia which was reported in an earlier paper (Goodman et al., 1965). Neither patient had any visual symptoms, and the vision in each was 20/20 OD and OS.

The fundus examination of the mother showed the typical findings of a choroideremia female carrier-i.e., moderate pigment clumping scattered throughout the fundus. Visual fields, dark-adaptation curves, final thresholds, and the electroretinogram were normal.

Follow-up examination 13 years later revealed no interim complaints or fundus changes. Fluorescein angiography indicated a choriocapillaris flush which was altered by the overlying pigment abnormalities. Areas of pigment loss resulted in a transmitted hyperfluorescence, the fine pigment accumulation gave the choriocapillaris flush phase a 'stippled' appearance, and areas of heavy pigment accumulation led to hypofluorescence. There was no evidence of choriocapillaris atrophy.

The initial picture of the 7-year-old son (case 11) was remarkably similar to that of his mother with pigment clumping scattered throughout the fundus. Peripheral visual fields were mildly constricted $(0 \cdot 25$ 

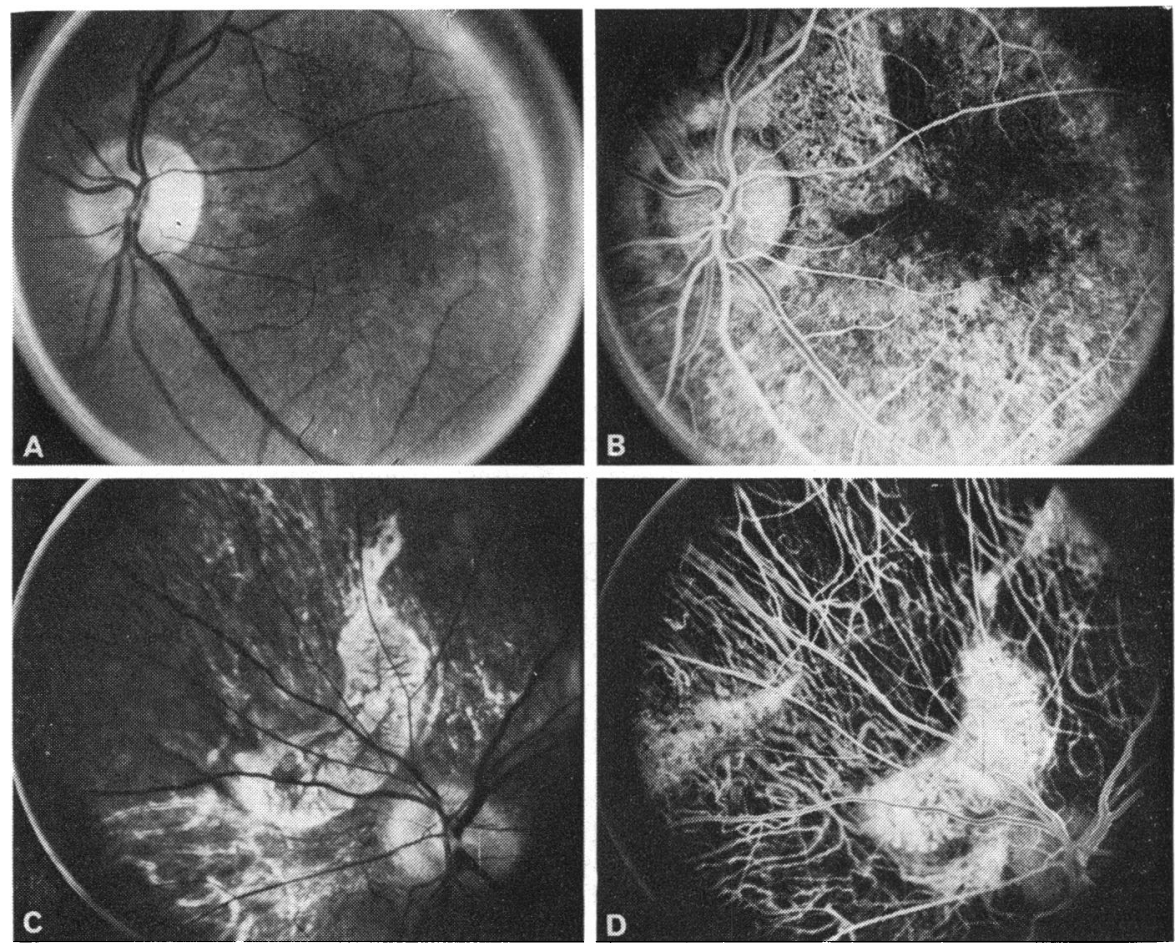

Fig. 7 (Noble, Carr, and Siegel) (case 10) ChOROIDEREMIA CARrIER. (A) Disc and macula of left eye showing pigment mottling. (B) Fluorescein angiography shows a normal choroidal fluorescence altered by the fine pigment mottling. Superiorly larger areas of pigment accumulation result in hypofluorescence. (Case 11) CHOROIDEREMIA. (C) Superonasal to the disc in the left eye. Adjacent to the disc is an area of intraretinal neovascular gliosis. There is diffuse thinning of the pigment epithelium and the large choroidal vessels are seen as white cords. In the area between 9 and $11 o^{\prime}$ 'clock the choroid appears normal. (D) Fluorescein angiography demonstrates a complete loss of choriocapillaris except for a small island. The 'normal appearing choroid is equally affected

$\mathrm{mm}^{2}$ white test object), final dark-adapted thresholds were elevated three log units, the amplitude of the photopic and scotopic electroretinogram response was reduced, and the electro-oculographic light rise was abnormal $(130 \%)$.

Thirteen years later the visual acuity remained relatively stable at 20/40 OD and 20/20 OS but there was a subjective loss in side vision and night vision. At this time the fundus had a diffuse thinning of the retinal pigment epithelium, some areas showing loss with 'sclerotic' choroidal vessels, other areas appearing granular with a normal choroid. Isolated areas of glial proliferation were observed.

Fluorescein angiography revealed a loss of choriocapillaris throughout the entire fundus, in both the funduscopically normal and 'sclerotic' appearing choroid. The choroidal vessels were easily observed and showed no abnormality. Isolated areas including the macula revealed choriocapillaris present.
The fluorescein angiogram in this female carrier showed the choriocapillaris to be present throughout the fundus. The choriocapillaris flush phase was altered by the overlying pigment epithelial changes and appeared as a hyperfluorescence, a hypofluorescence, or as a fine stippling, the appearance depending on the nature of the change. Therefore, the only disturbance appreciated in the carrier female on fluorescein were the pigmentary abnormalities observed on fundus examination.

Krill (1969) noted that the carrier female and early affected males had a similar pigmentary mottling. Since fluorescein angiography of the carrier showed only an abnormality in the pigment epithelium, he reasoned that the primary defect was in the pigment epithelium. However, atrophy of the choriocapillaris that occurs early in choroideremia cannot always be easily visualised on fundus examination alone.

Thus, in the affected male (case 11) there were areas 
of the fundus which appeared similar to the female carrier-i.e., pigment mottling and normal choroidbut fluorescein angiography clearly showed complete choriocapillaris loss. Therefore, despite the similarities of the early affected male and the female carrier, the loss of choriocapillaris evident on fluorescein angiography may explain the difference in the visual function and prognosis of the disease.

\section{Discussion}

The hereditary choroidal dystrophies are a heterogeneous group of diseases in which an aetiology is not known. The common feature of these disorders is a loss of the choriocapillaris in the early stages which progresses to involve the retinal pigment epithelium, the photoreceptor layer, and in some cases the entire choroid. Whether the primary abnormality is in the choriocapillaris or in some other tissue has not been determined.

Certain of these dystrophies are generalised and progressive (choroideremia, generalised choroidal dystrophy) whereas others are localised, either remaining geographically confined (central areolar choroidal dystrophy) or progressively expanding (peripapillary choroidal dystrophy and gyrate atrophy) to involve more and more of the fundus.

The earliest ophthalmoscopic manifestations may range from subtle to marked alterations in the pigment epithelium. Mild pigment granularity, a tapetal 'sheen', pigment epithelial atrophy, or pigment accumulation may singly or in combination represent the first signs. These may be difficult to distinguish from certain other hereditary retinal degenerations or postinflammatory changes. Similarly in the end stages of the choroidal dystrophies the loss of retinal and choroidal tissue, exposure of the sclera, heavy pigment accumulation, and vascular changes cannot be easily differentiated from other degenerative and inflammatory conditions.

The role of fluorescein angiography in the study of the hereditary choroidal dystrophies is relatively new and seems to give useful information in diagnosing and understanding these disease entities. In terms of diagnosis the most helpful situation is in the early stages of the disease when the choroid appears normal and only the pigment epithelium is altered. In the hereditary choroidal dystrophies the absent choriocapillaris will be readily apparent on fluorescein angiography. During the choroidal flush phase the homogeneous dense background fluorescence due to choriocapillaris filling and subsequent leakage is absent and the large choroidal vessels remain visible throughout the entire sequence. When the pigment epithelium alone is involved without choriocapillaris disease, a normal choriocapillaris phase is seen though its appearance may be altered by the overlying pigment to show hyper- or hypofluorescence.

Fluorescein studies clearly distinguish between the generalised and local dystrophies. In central and peripapillary choroidal dystrophy as well as in gyrate atrophy there is a normal choriocapillaris flush adjacent to the involved tissue. In choroideremia and generalised choroidal dystrophy the choriocapillaris is involved throughout the retina, even though some areas may appear less involved than others.

With regard to an understanding of the aetiology, the role of fluorescein angiography is less obvious. Certainly the loss of the choriocapillaris is a very early sign, but so are the pigment abnormalities. A causal relationship has not been established. It is known, however, that in the early stages of many of the tapeto-retinal degenerations and certain hereditary macular degenerations (Best's, Stargardt's) fluorescein angiography does not indicate a choriocapillaris abnormality. This is one of the reasons such diseases are placed in the category of primary pigment epithelial-photoreceptor disorders. It is only in the very advanced stages that the choroidal vessels may be affected in these degenerations. By comparison, in the hereditary choroidal dystrophies the early loss of the choriocapillaris is seemingly of primary importance in the pathogenesis of these diseases.

In a discussion of these disorders it is important to clarify several facets of the problem. It would be virtually impossible to state with any degree of finality where the initial inciting abnormality begins. It is, however, difficult to imagine that there would be a situation in which there would be a choriocapillaris abnormality in which the dependent adjacent pigment epithelium was not involved. Yet there are many cases in which the converse can be seen-i.e., abnormalities of the pigment epithelium with a normal choriocapillaris. Thus, a hypothesis could be set forth denoting those diseases which in the early stages show an associated choriocapillaris abnormality and to theorise that this is the initial abnormality. Such cases would be under the broad umbrella of the choroidal dystrophies.

The authors are indebted to Mr Walter Lentschner, photographer, and Miss Sylvia Kwastel, secretary and typist.

\section{References}

Carr, R. E. (1965). Archives of Ophthalmology, 73, 32.

Carr, R. E., Ripps, H., Siegel, I. M., and Weale, R. A. (1966a). Investigative Ophthalmology, 5, 497.

Carr, R. E., Gouras, P., and Gunkel, R. D. (1966b). Archives of Ophthalmology, 75, 171. 
Carr, R. E., Mittl, R. N., and Noble, K. G. (1975). Transactions of the American Academy of Ophthalmology and Otolaryngology, 79, 796.

Curry, H. F., and Schonberg, S. S. (1969). Archives of Ophthalmology, 81, 177.

Duke-Elder, S. (1966). System of Ophthalmology, vol. 9, 'Diseases of the Uveal Tract', p. 700, St. Louis, C. V. Mosby.

Franceschetti, A., Francois, J., and Babel, J. (1974). Choroioretinal Heredo-degenerations, pp. 495, 497, 505, 506, 512, 569, 570, 575, 575. Springfield, Ill. Charles C Thomas.

Fraser, G. R., and Friedmann, A. I. (1968). British Medical Journal, 2, 732.

Goodman, G., Ripps, H., and Siegel, I. M. (1965). Archives of Ophthalmology, 73, 387.

Harris, G. S., and Miller, J. R. (1968). Archives of Ophthalmology, 80, 423.

Krill, A. E. (1969). Transactions of the American Ophthalmological Society, 67, 535.
Krill, A. E., and Archer, D. (1971). American Journal of Ophthalmology, 72, 562.

Kurstjens, J. H. (1965). Documenta Ophthalmologica, 19, 1.

McCulloch, J. C., and McCulloch, R. J. P. (1948). Transactions of the American Academy of Ophthalmology and Otolaryngology, 52, 160.

McKay, R. H., and Spivey, B. E. (1962). Archives of Ophthalmology, 67, 727

Sandvig, K. (1955). Acta Ophthalmologica, 33, 71.

Sorsby, A., and Crick, R. P. (1953). British Journal of Ophthalmology, 37, 129.

Sorsby, A., and Davey, L. B. (1955). British Journal of Ophthalmology, 39, 257.

Svensson, K. (1939). Acta Ophthalmologica, 17, 73.

Waardenberg, P. J. (1939). Nederlandsch Tijdschrift voor Geneeskunde, 83, 4978.

Waardenberg, P. J. (1952). Journal de Génétique Humaine, 1, 83. 